\title{
Scaling the immune incline in PDAC
}

\author{
Luis A. Rojas (10 ${ }^{1}$ and Vinod P. Balachandran (10) ${ }^{1,2 凶}$
}

Pancreatic ductal adenocarcinoma (PDAC) reflects the current challenge for immuno-oncology: to develop new drugs for cancers that are resistant to current immunotherapies. Here, we argue for a need to use patients to uncover new strategies that activate anti-tumour immunity.

Clinical evidence that immune checkpoint inhibitors (ICIs) induce durable responses in patients with cancer has established a positive 'litmus test' for cancer immunotherapy - drugs that target host immunity can control, shrink and even eradicate human cancer ${ }^{1}$. This remarkable proof, coupled with the burgeoning realization that the immune system controls cancer through a myriad of different ways that could be harnessed as therapies, has positioned immuno-oncology to lead the next cancer therapy revolution. To fulfill this promise, however, cancer immunotherapy must meet its next critical challenge - it must deliver new drugs to treat more cancers. Pancreatic ductal adenocarcinoma (PDAC), among the most ICI-resistant cancers ${ }^{1}$, is a microcosm of this challenge.

Conceptually, there seem to be two immunological barriers to this challenge in cancer: insufficient immune activation and excess immune suppression. In as much as immune responses to tumours progress sequentially that is, antigen presenting cells (APCs) capture tumour antigens to prime $\mathrm{T}$ cells that traffic to tumours and express lytic molecules that trigger counter-regulatory suppressive pathways - one can reasonably conclude that tumour immune activation precedes suppression. Thus, as PDAC expresses fewer transcripts of genes that estimate $\mathrm{T}$ cell infiltration $(C D 8)$, activation (PRF1, GZMB, $I F N G$ ) and suppression (CTLA4, PD1, PDL1, LAG3, $I D O 1)^{2}$ than ICI-responsive tumours, it follows that the dominant defect in PDAC seems to be insufficient activation. This conclusion aligns with observations that, compared with ICI-responsive tumours such as melanoma or mismatch repair-deficient (MMRd) cancers, PDAC and other ICI-resistant cancers are less antigenic; they have $\sim 10-20$-fold fewer somatic mutations ${ }^{1}$, which are key drivers of both endogenous ${ }^{3}$ and ICI-induced anti-tumour immune responses ${ }^{1}$. Thus, PDAC appears to halt earlier on a 'cancer immune incline' compared to ICI-responsive tumours (FIG. 1). PDAC, therefore, has lower immune activation 'potential energy' and, accordingly, blockade of suppression alone is insufficient to generate clinical responses ${ }^{1}$. By contrast, ICI-responsive tumours such as melanoma and MMRd cancers acquire a higher immune activation potential energy as they progress higher up the incline. Thus, release of suppression is sufficient to induce clinical activity. Although largely conceptual, the cancer immune incline can provide a framework to rationally design immunotherapies for different tumours. To scale this incline in PDAC we propose there is an increased need for therapies that activate immunity ${ }^{4}$.

Although mouse models have uncovered important insights into the genetic drivers of PDAC carcinogenesis, deep dives into human PDAC immunobiology have revealed important distinctions between PDAC in humans and mice. First, mutations appear to be more important drivers of anti-tumour immunity in human PDAC than in genetically engineered mouse models (GEMMs). Human PDACs harbour putative immunogenic mutations ( $\sim 30$ neoantigens per tumour) that confer strong endogenous immunity when sufficiently immunogenic ${ }^{5}$ and even stronger immunity that render PDACs ICI-responsive when sufficiently numerous (as in MMRd PDACs) ${ }^{6}$. However, immunity against GEMM-derived PDAC is not directed at neoantigens as there are few ( $\sim 0-11$ per tumour $)^{7}$. Second, immunity against human PDAC varies in strength between patients, possibly driven by differences in antigens, tumour microenvironments, polymorphic combinations of somatic genotype, host haplotype and inherited or acquired ${ }^{8}$ factors, which all appear to influence immunity to different degrees. It is clear this heterogeneity is not captured by current mouse models. Thus, investigation should emphasize reverse translation - discover in patients and dissect mechanisms in mice. In this regard, the study of biological extremes in patients with PDAC, such as exceptional responders to therapies and long-term survivors ${ }^{5}$, represent unique discovery tools. The role of the gut microbiota ${ }^{8}$, novel anti-tumour innate immune cells ${ }^{9}$, and PD1 inhibitors in rare PDAC subsets ${ }^{6}$ are examples of novel areas of scientific and clinical focus in PDAC that originated from this approach.

From an immune perspective, accelerating the pace of discovery in patients requires concerted efforts to collect, analyze and disseminate immunological data obtained from patients with PDAC. This approach is particularly relevant as immunological data from human PDAC remain scarce, many labs have limited access to patient material and sample acquisition is challenging given that PDACs are small and often in poorly accessible anatomic locations. However, multiple groups have 


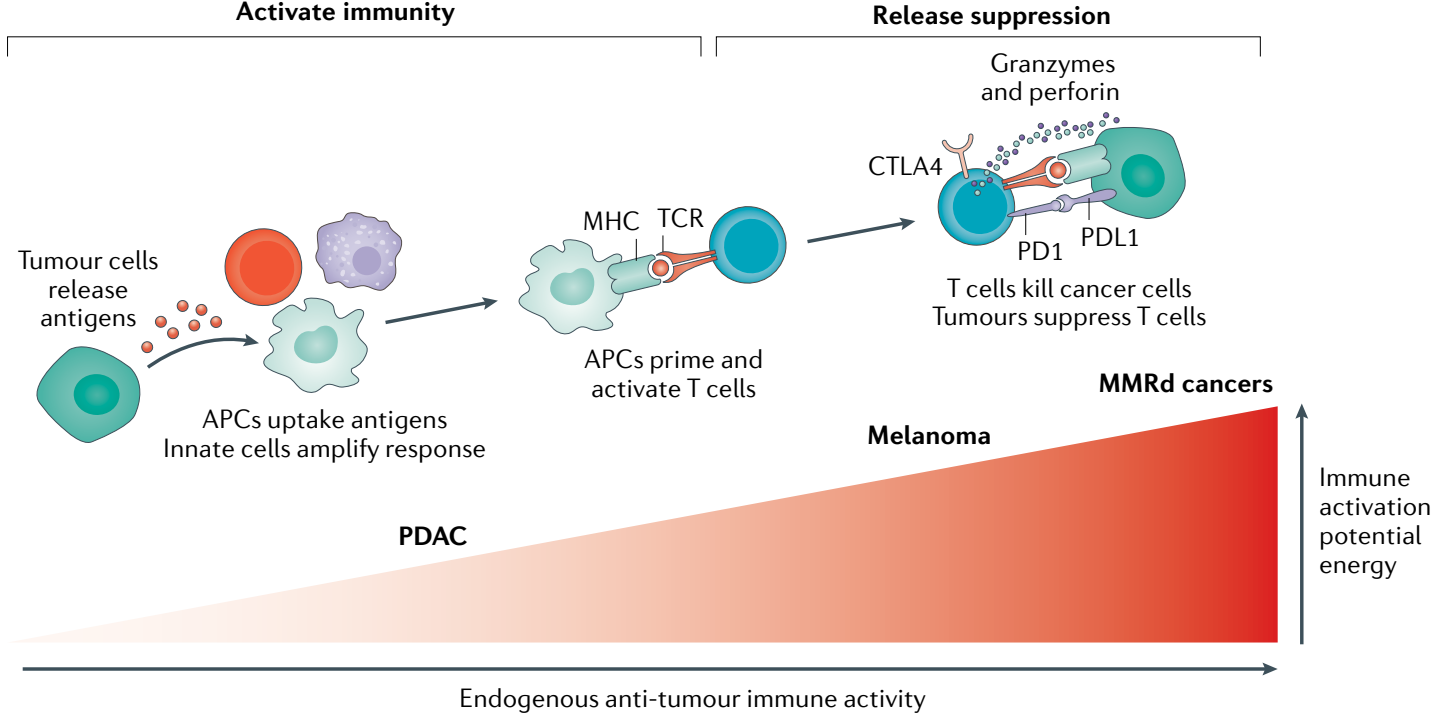

Fig. 1 | The cancer immune incline. Pancreatic ductal adenocarcinoma (PDAC) arrests earlier on a 'cancer immune incline' than immune checkpoint inhibitor (ICl)-responsive tumours such as melanoma or mismatch repair deficient (MMRd) tumours. PDAC has lower immune activation 'potential energy', and blockade of suppression alone with ICls targeting PD1, PDL1 or CTLA4 is insufficient to generate a clinical response. Thus, therapies that activate immunity are required. APC, antigen presenting cell; TCR, T-cell receptor. $\odot 2021$ Memorial Sloan Kettering Cancer Center. All rights reserved.

now demonstrated that human PDAC genomes, exomes and transcriptomes can be captured at large scales ${ }^{10}$. These advances, coupled with the expanding repertoire of computational tools that use such high-dimensional sequencing data to visualize the tumour immune environment, provide blueprints to rapidly advance our understanding of human PDAC immunobiology. Such efforts will require intra-institutional commitments to prioritize, collect and study samples from patients with PDAC, coupled with inter-institutional, national and global efforts to disseminate data broadly and freely. One solution is to create a central human PDAC data repository (such as the pan-cancer genomics cBioPortal) of samples collected, not only prospectively from patients with PDAC undergoing standard treatment, but also from those treated on clinical trials, who are often the maximally studied, yet most scientifically data-opaque patient population. The ability of free, near-immediate data access to accelerate the pace of discovery and translation is a critical lesson that has emerged from the coronavirus disease 2019 pandemic, and we must now harness this approach for PDAC.

Although testing drugs from the metastatic to the early disease setting is ideal for cytotoxic and targeted therapies, seeking immunotherapy signals with a similar approach requires caution. Unlike therapies that target cancer cells, immunotherapies that target host cells are more susceptible to changes in the immunological state of the host. This situation might even be more true for low immune-potential tumours such as PDAC, where the metastatic setting might place additional immunological hurdles along the cancer immune incline to obscure signs of drug activity. This understanding might require a rethink to our approach of testing drugs sequentially from metastatic to early disease, and to instead test more immunotherapies in earlier cancer settings. Such a reprioritization, along with early immunological endpoints to identify if drugs are on or off target, can facilitate early decisions to efficiently select promising immunotherapies for larger studies. This approach, coupled with increased efforts to iteratively learn from patients, increase data sharing and enable free data access, could fuel discovery at a much larger scale.

Despite positive correlations between heightened immune activity and improved long-term survival in $\mathrm{PDAC}^{5}$, whether immunity can be harnessed as a therapy for PDAC has been a lingering question. The ability of a PD1 blocking antibody to induce a $>60 \%$ response in patients with MMRd PDACs ${ }^{6}$ has now provided a definitive proof-of-principle. Immunotherapy is possible for PDAC - we must now shift our focus from 'if' it can be harnessed, to 'how'.

1. Yarchoan, M., Hopkins, A. \& Jaffee, E. M. Tumor mutational burden and response rate to PD-1 inhibition. N. Engl. J. Med. 377 , 2500-2501 (2017).

2. Bailey, P. et al. Exploiting the neoantigen landscape for immunotherapy of pancreatic ductal adenocarcinoma. Sci. Rep. 6, $35848(2016)$

3. Rooney, M. S., Shukla, S. A., Wu, C. J., Getz, G. \& Hacohen, N. Molecular and genetic properties of tumors associated with local immune cytolytic activity. Cell 160, 48-61 (2015).

4. Vonderheide, R. H. CD40 Agonist antibodies in cance immunotherapy. Annu. Rev. Med. 71, 1-12 (2019).

5. Balachandran, V. P. et al. Identification of unique neoantigen qualities in long-term survivors of pancreatic cancer. Nature 551, 512-516 (2017).

6. Le, D. T. et al. Mismatch repair deficiency predicts response of solid tumors to PD-1 blockade. Science 357, 409-413 (2017).

7. Evans, R. A. et al. Lack of immunoediting in murine pancreatic cancer reversed with neoantigen. JCl insight 1, (2016).

8. Riquelme, E. et al. Tumor microbiome diversity and composition influence pancreatic cancer outcomes. Cell 178, 795-806.e12 (2019).

9. Moral, J. A. et al. ILC2s amplify PD-1 blockade by activating tissue-specific cancer immunity. Nature 579, 790-796 (2020).

10. Bailey, P. et al. Genomic analyses identify molecular subtypes of pancreatic cancer. Nature 531, 47-52 (2016).

\section{Acknowledgements}

This work was supported by a Damon Runyon Clinical Investigator Award to V.P.B and an NIH U01 CA224175 to V.P.B.

\section{Competing interests}

The authors declare no competing interests. 